\title{
Is miRNA-375 a promising biomarker for early detection and monitoring of patients with type 2 diabetes?
}

Dimitrios loannis Patoulias ${ }^{1,2}$

${ }^{1}$ Department of Internal Medicine, General Hospital of Veria, Veria, Greece ${ }^{2}$ Second Propedeutic Department of Internal Medicine, Aristotle University of Thessaloniki, General Hospital Hippokration, Thessaloniki, Greece

Submitted: 22 April 2018

Accepted: 2 August 2018

Arch Med Sci Atheroscler Dis 2018; 3: e119-e122

DOI: https://doi.org/10.5114/amsad.2018.78775

Copyright $\odot 2018$ Termedia \& Banach

\begin{abstract}
miRNAs are small, non-coding RNAs, functioning as negative suppressors of target gene expression. A significant proportion of the transcriptome is subject to miRNA modulation. A single miRNA determines the expression of hundreds of genes, while miRNAs are relatively stable in biological fluids. Thus, they have attracted scientific interest regarding their use as biomarkers for several diseases. miRNA-375 mainly influences $\beta$-cell function and insulin secretion. Several studies, primarily experimental, have assessed its role as a biomarker in type 2 diabetes, while recently obtained human evidence supports this potential role. Besides its diagnostic potential, miRNA-375 may also have therapeutic implications. In view of the growing epidemic of type 2 diabetes, there is an unmet need for identification of biomarkers for early recognition and monitoring of these patients. Long-term, prospective human studies are required to elucidate whether miRNA-375 can evolve as a key player in diagnosis and prognosis of type 2 diabetes.
\end{abstract}

Key words: miRNA-375, type 2 diabetes, $\beta$-cell, biomarker.

\section{Introduction}

miRNAs are endogenous, single-stranded, non-coding RNAs, 18-25 nucleotides in length, which were first identified in the nematode Caenorhabditis elegans in 1993 [1, 2]. They regulate negatively, or repress, target gene expression. Specifically, they bind with the 3'-untranslated region ( $3^{\prime}$-UTR) of the target mRNA via imperfect base pairing, inducing mRNA degradation, deadenylation or translational inhibition [3]. The degree of complementarity between miRNAs and the 3'-UTR region is crucial for the regulation of target gene expression [4].

miRNAs are present in circulation due to leakage from damaged or dead cells. In human blood, they are packaged into extracellular vesicles, and they are also associated with high-density lipoprotein (HDL) or are even bound by RNA-binding protein argonaute-2. Consequently, their degradation is prevented, leading to extended circulation times $[5,6]$. Thus, they are an attractive and developing cluster of biomarkers for several diseases, with ongoing experimental and human studies assessing their potential diagnostic and prognostic utility in clinical practice.

Type 2 diabetes mellitus (T2DM) accounts for more than $90 \%$ of all cases of diabetes. Overall, it affects more than 350 million patients, while

\author{
Corresponding author: \\ Dimitrios Ioannis Patoulias \\ Department of \\ Internal Medicine \\ General Hospital \\ of Veria \\ 3 B M. Alexandrou St \\ 57010 Thessaloniki, Greece \\ Phone: +30 6946900777 \\ E-mail: dipatoulias@gmail. \\ com
}


its prevalence is predicted to exceed 550 million patients by 2030 [7-9].

From a pathophysiological view, T2DM is multifactorial. Prolonged hyperglycemia / hyperlipemia (glucolipotoxicity) induces progressive $\beta$-cell dysfunction and finally $\beta$-cell apoptosis, leading to impaired insulin production $[10,11]$.

There is an unmet need for early recognition of type 2 diabetic patients, in order to prevent disease progression and the development of complications, along with the identification of novel therapeutic targets.

\section{miRNA-375: function and targets}

miRNA-375 is predominantly expressed in $\beta$-cells, being well described in terms of its association with $\beta$-cell function and insulin secretion [12]. It targets the MTPN gene encoding myotrophin, a regulatory protein for insulin secretion via exocytosis of insulin granules, and PKD1, a gene involved in activation of the PI3-Akt pathway. Overexpression of miRNA-375 finally leads to inactivation of the aforementioned pathway, and subsequently to decreased $\beta$-cell mass, expression of the insulin gene and, finally, insulin release [13].

According to the classic study by Poy et al. [14], miRNA-375 deletion in mice influences not only $\beta$-cell mass, but also $\alpha$-cell mass, while Joglekar and colleagues demonstrated that miRNA-375 expression is high during pancreatic islet development [15]. Overall, miRNA-375 seems to be crucial for formation of the $\beta$-cell phenotype [16].

Target genes of miRNA-375 can be classified into three groups. The first group consists of pancreatic development related genes, such as Sox17, Sox9, GATA6, Hnf1 $\beta$ and Pax6. The second group includes cell growth and proliferation associated genes, such as Cav1, Id3, Smarca2, Aifm1, Rasd1, Rgs16, Eef1e1, C1qbp, HuD, and Cadm, while the third group includes insulin secretion related genes, mainly MTPN and PKD1 [17].

\section{miRNA-375: diagnostic and prognostic implications}

A recent meta-analysis conducted by Zhu and Leung demonstrated that miRNA-375 was the most upregulated miRNA in human profiling studies involving type 2 diabetic patients, constituting a novel circulating biomarker for T2DM [18]. Another meta-analysis by Villard et al. confirmed the above results, documenting that miRNA-375 displayed the highest fold changes between diabetic and control subjects [19]. Indeed, the authors estimate that the different levels observed between newly diagnosed and long-term diabetic patients may be indicative of pathophysiological alterations occurring early after the onset of the disease [19].
Until now, several published experimental and human studies have highlighted the potential role of miRNA-375 as a biomarker in T2DM, as it possibly reflects the ongoing $\beta$-cell death during the disease course [20-23]. Zhao et al. correlated the levels of pancreatic expression of miRNA-375 with the severity of pancreatic islet amyloid formation and the induced $\beta$-cell deficit, a finding which can unravel new pathophysiological mechanisms in T2DM [24].

As far as the early predictive value of miRNA-375 in T2DM is concerned, Al-Muhtaresh and Al-Kafaji attempted to shed light on whether this promising biomarker can detect prediabetes and T2DM [25]. The researchers enrolled 30 subjects with prediabetes (either impaired fasting glucose or impaired glucose tolerance), 30 subjects with T2DM and 30 controls. miRNA-375 expression was 3-fold higher in subjects with prediabetes and 5.9-fold higher in subjects with T2DM, compared to controls $(p<0.001)$. A statistically significant difference was also observed between subjects with prediabetes and T2DM, as in the latter group miRNA-375 expression was 2-fold higher $(p=0.04)$. Multivariate logistic regression analysis using the prediabetes group as a reference revealed that miRNA-375 was significantly associated with T2DM, after adjustment for age, sex, BMI, lipid profile and mean blood pressure. In both groups, prediabetes and T2DM, miRNA-375 displayed a significant positive correlation with well-established measures of glycemic control, mainly fasting glucose and $\mathrm{HbA}_{1 c}$, along with the oral glucose tolerance test (OGTT). Notably, this study included a ROC analysis, in order to determine whether miRNA-375 can discriminate subjects with prediabetes or T2DM from controls: this promising biomarker exhibited an area under the curve (AUC) of 0.76 for discrimination between prediabetes patients and controls $(p=0.001)$, of 0.77 for discrimination between patients with T2DM and controls $(p<0.001)$ and of 0.78 when discriminating patients with T2DM from those with prediabetes $(p=0.047)$. Meticulous interpretation of the derived data leads to the conclusion that miRNA-375 is implicated in the pathogenesis and the progression of T2DM, and thus it constitutes a promising biomarker for early detection and monitoring of these patients [25].

It is noteworthy that, in their cross-sectional pilot study, Seyhan et al. did not observe a statistically significant difference in circulating levels of miRNA-375 between subjects with prediabetes or T2DM and healthy controls [26]. The researchers also found that circulating levels of miRNA-375 were significantly higher in subjects with type 1 diabetes mellitus (T1DM) compared to controls; thus they question whether miRNA-375 is not just a biomarker of $\beta$-cell injury and function loss, but a marker indicative of autoimmunity as well [26]. 
In a recently published study by Saravanan et al. involving 12 patients with chronic pancreatitis who underwent total pancreatectomy and islet autotransplantation, it was found that miRNA-375 expression was significantly and positively correlated with glycemic control and requirements in exogenous insulin reposition at 6 months post-transplantation (for $\mathrm{HbA}_{1 c}: r=0.67$, $p=0.04$ and for insulin requirements: $r=0.87$, $p=0.003$ ) [27]. Those findings are suggestive of the potential role of miRNA-375 as a $\beta$-cell biomarker. Previous work of the same research team revealed the promising role of miRNA-375 in predicting post-transplantation graft function and overall metabolic function in the same study group [28].

However, there is insufficient evidence regarding the association between miRNA-375 expression and the development of diabetic micro- and macrovascular complications. Li et al. formulated the hypothesis that miRNA-375 circulating levels are decreased in early stages of diabetic nephropathy, as it binds to their target tissues, in order to maintain body homeostasis [29].

Regarding monitoring of the administered antidiabetic treatment, there is also limited evidence to support the role of miRNA-375. Xu et al. recently published the results of their study in a mouse model, demonstrating that liraglutide treatment increased miRNA-375 and cell apoptosis in pancreatic $\alpha$-cells via inhibition of the CAMP-PKA signaling pathway, while it activated the same pathway in $\beta$-cells, resulting in downregulation of miRNA-375 [30]. In an experimental mouse model of high fat diet induced obesity, Lee et al. demonstrated that exenatide, another GLP-1 receptor agonist, ameliorates serum levels of miRNA-375, reflecting the improvement in insulin sensitivity. Thus, they concluded that miRNA-375 can be useful as a biomarker for monitoring the response to exenatide treatment, indicating changes in insulin sensitivity [31].

Besides being an indicator of $\beta$-cell dysfunction and $\beta$-mass loss in patients with T2DM, miRNA-375 could also serve as a therapeutic target for prevention of disease progression [32]. There is also significant interest in the results of the study conducted by Lahmy et al., who documented that miRNA-375 can promote pancreatic differentiation of human induced pluripotent stem cells, and thus can be used for $\beta$-cell replacement therapy in diabetic patients [33]. In their recently published experimental study, Song et al. documented that miRNA-375 could serve as an ideal biomarker, not only for detection of $\beta$-cell death, but also for testing novel antidiabetic drugs featuring cytoprotective effects on $\beta$-cells [34]. Keller et al. also described the potential utility of antagonizing miRNA-375 in T2DM patients, which can facilitate the antidiabetic effects of exendin-4, administered pre-meal [35].

\section{Conclusions}

It is crucial to quantify the impact of $\beta$-cell failure on the development and progression of T2DM. miRNA-375 may serve as a promising biomarker for early recognition of diabetic patients, which will contribute to the prevention of complications, along with monitoring of the applied treatment.

Of course, the association of miRNA-375 expression levels with well-established parameters of glycemic control, insulin resistance and $\beta$-cell function, namely $\mathrm{HbA}_{1 \mathrm{c}}, \mathrm{FPG}, \mathrm{HOMA}-\mathrm{IR}$ and HOMA- $\beta$, may offer the perspective of development of novel diagnostic panels, with great applicability for both early diagnosis and monitoring of these patients.

However, we admit that until now there is not sufficient evidence from human studies to establish miRNA-375 as a new biomarker in T2DM. Further, prospective, long-term human studies are required to elucidate this realistic hypothesis.

\section{Conflict of interest}

The author declares no conflict of interest.

\section{References}

1. Lee RC, Feinbaum RL, Ambros V. The C. elegans heterochronic gene lin-4 encodes small RNAs with antisense complementarity to lin-14. Cell 1993; 75: 843-54.

2. Wightman B, Ha I, Ruvkun G. Posttranscriptional regulation of the heterochronic gene lin-14 by lin- 4 mediates temporal pattern formation in C. elegans. Cell 1993; 75: 855-62.

3. Guo H, Ingolia NT, Weissman JS, Bartel DP. Mammalian microRNAs predominantly act to decrease target mRNA levels. Nature 2010; 466: 835-40.

4. Kim VN, Han J, Siomi MC. Biogenesis of small RNAs in animals. Nature Rev Mol Cell Biol 2009; 10: 126-39.

5. Turchinovich A, Weiz L, Langheinz A, Burwinkel B. Characterization of extracellular circulating micro-RNA. Nucleic Acids Res 2011; 39: 7223-33.

6. Arroyo JD, Chevillet JR, Kroh EM, et al. Argonaute2 complexes carry a population of circulating microRNAs independent of vesicles in human plasma. Proc Natl Acad Sci U S A 2011; 108: 5003-8.

7. Gao HX, Regier EE, Close KL. International Diabetes Federation World Diabetes Congress 2015. J Diabetes 2016; 8: 300-2.

8. Whiting DR, Guariguata L, Weil C, Shaw J. IDF Diabetes Atlas: global estimates of the prevalence of diabetes for 2011 and 2030. Diabetes Res Clin Pract 2011; 94: 311-21.

9. Chen L, Magliano DJ, Zimmet PZ. The worldwide epidemiology of type 2 diabetes mellitus: present and future perspectives. Nat Rev Endocrinol 2012; 8: 228-36.

10. Leibowitz G, Kaiser N, Cerasi E. Beta-cell failure in type 2 diabetes. J Diabetes Investig 2011; 2: 82-91.

11. Maedler K, Donath MY. Beta-cells in type 2 diabetes: a loss of function and mass. Horm Res 2004; 62 Suppl 3: 67-73. 
12. van de Bunt M, Gaulton KJ, Parts L, et al. The miRNA profile of human pancreatic islets and beta-cells and relationship to type 2 diabetes pathogenesis. PLoS One 2013; 8: e55272.

13. Bhatia P, Raina S, Chugh J, Sharma S. miRNAs: early prognostic biomarkers for type 2 diabetes mellitus? Biomark Med 2015; 9: 1025-40.

14. Poy MN, Hausser J, Trajkovski M, et al. miR-375 maintains normal pancreatic alpha- and beta-cell mass. Proc Natl Acad Sci USA 2009; 106: 5813-8.

15. Joglekar MV, Joglekar VM, Hardikar AA. Expression of islet-specific microRNAs during human pancreatic development. Gene Expr Patterns 2009; 9: 109-13.

16. Eliasson L. The small RNA miR-375 - a pancreatic islet abundant miRNA with multiple roles in endocrine beta cell function. Mol Cell Endocrinol 2017; 456: 95-101.

17. Li X. MiR-375, a microRNA related to diabetes. Gene 2014; 533: 1-4.

18. Zhu H, Leung SW. Identification of microRNA biomarkers in type 2 diabetes: a meta-analysis of controlled profiling studies. Diabetologia 2015; 58: 900-11.

19. Villard A, Marchand L, Thivolet C, Rome S. Diagnostic value of cell-free circulating microRNAs for obesity and type 2 diabetes: a meta-analysis. J Mol Biomark Diagn 2015; 6: pii: 251.

20. Delic D, Eisele C, Schmid R, Luippold G, Mayoux E, Grempler R. Characterization of micro-RNA changes during the progression of type 2 diabetes in Zucker diabetic fatty rats. Int J Mol Sci 2016; 17: pii: E665.

21. Higuchi C, Nakatsuka A, Eguchi J, et al. Identification of circulating miR-101, miR-375 and miR-802 as biomarkers for type 2 diabetes. Metabolism 2015; 64: 489-97.

22. Sun K, Chang X, Yin L, et al. Expression and DNA methylation status of microRNA-375 in patients with type 2 diabetes mellitus. Mol Med Rep 2014; 9: 967-72.

23. Wang X, Chang X, Li J, Yin L, Sun K. DNA methylation of microRNA-375 in impaired glucose tolerance. Exp Ther Med 2014; 8: 775-80.

24. Zhao H, Guan J, Lee HM, et al. Up-regulated pancreatic tissue microRNA-375 associates with human type 2 diabetes through beta-cell deficit and islet amyloid deposition. Pancreas 2010; 39: 843-6.

25. Al-Muhtaresh HA, Al-Kafaji G. Evaluation of two-diabetes related microRNAs suitability as earlier blood biomarkers for detecting prediabetes and type 2 diabetes mellitus. J Clin Med 2018; 7: pii: E12.

26. Seyhan AA, Nunez Lopez YO, et al. Pancreas-enriched miRNAs are altered in the circulation of subjects with diabetes: a pilot cross-sectional study. Sci Rep 2016; 6: 31479.

27. Saravanan PB, Kanak MA, Chang CA, et al. Islet damage during isolation as assessed by miRNAs and the correlation of miRNA levels with posttransplantation outcome in islet autotransplantation. Am J Transplant 2018; 18: 982-9.

28. Yoshimatsu G, Takita M, Kanak MA, et al. MiR-375 and miR-200c as predictive biomarkers of islet isolation and transplantation in total pancreatectomy with islet autotransplantation. J Hepatobiliary Pancreat Sci 2016; 23 585-94.

29. Li Y, Xu X, Liang Y, et al. miR-375 enhances palmitate-induced lipoapoptosis in insulin-secreting NIT-1 cells by repressing myotrophin (V1) protein expression. Int J Clin Exp Pathol 2010; 3: 254-64.

30. $\mathrm{Xu} \mathrm{X,} \mathrm{Chen} \mathrm{J,} \mathrm{Hu} \mathrm{L,} \mathrm{et} \mathrm{al.} \mathrm{Liraglutide} \mathrm{regulates} \mathrm{the} \mathrm{via-}$ bility of pancreatic alpha-cells and pancreatic beta-cells through cAMP-PKA signal pathway. Life Sci 2018; 195 87-94.

31. Lee IS, Park KC, Yang KJ, et al. Exenatide reverses dysregulated microRNAs in high-fat diet-induced obese mice. Obes Res Clin Pract 2016; 10: 315-26.

32. Yang Y, Xiao L, Li J, Kanwar YS, Liu F, Sun L. Urine miRNAs: potential biomarkers for monitoring progression of early stages of diabetic nephropathy. Med Hypotheses 2013; 81: 274-8.

33. Lahmy R, Soleimani M, Sanati MH, Behmanesh M, Kouhkan F, Mobarra N. MiRNA-375 promotes beta pancreatic differentiation in human induced pluripotent stem (hiPS) cells. Mol Biol Rep 2014; 41: 2055-66.

34. Song I, Roels S, Martens GA, Bouwens L. Circulating microRNA-375 as biomarker of pancreatic beta cell death and protection of beta cell mass by cytoprotective compounds. PLoS One 2017; 12: e0186480.

35. Keller DM, Clark EA, Goodman RH. Regulation of microRNA-375 by CAMP in pancreatic beta-cells. Mol Endocrinol 2012; 26: 989-99. 